\title{
Using Economic Games to Investigate the Neural Substrates of Cognitive Processes
}

\author{
Masao Nagatsuka ${ }^{1}$, Hideo Shinagawa ${ }^{1, *}$, Yoshiyaka Okano ${ }^{2}$, Yuri Kitamura ${ }^{3}$, Tatsuyoshi Saijo ${ }^{2}$ \\ ${ }^{1}$ Research Center for Behavioral Economics, The Institute of Social and Economic Research (ISER), Osaka University, Osaka, Japan \\ ${ }^{2}$ Department of Management, Kochi University of Technology, Kochi, Japan \\ 3Department of Social and Environmental Medicine, Graduate School of Medicine, Osaka University, Osaka, Japan \\ *Corresponding author: sinagawa@iser.osaka-u.ac.jp
}

Received November 12, 2013; Revised November 20, 2013; Accepted December 03, 2013

\begin{abstract}
Our study has shown that changes can be induced in human decision-making and emotions by using the PD and PDAS games. This suggests that economic games may provide a way to study cognitive functions in detail by analyzing the structure of the economic games using game theory. In addition, the combined use of economic games and neuroimaging techniques such as fNIRS, fMRI, and MEG may capture and quantify neural the substrates of various cognitive processes in a clinical setting. They might be effective in extracting human emotional ups and downs in the process of the decision-making. Further studies of economic games for patients with mental illness or brain damage are needed to explore this approach further.
\end{abstract}

Keywords: economic games, prisoners' dilemma, fNIRS

Cite This Article: Masao Nagatsuka, Hideo Shinagawa, Yoshiyaka Okano, Yuri Kitamura, and Tatsuyoshi Saijo, "Using Economic Games to Investigate the Neural Substrates of Cognitive Processes.” American Journal of Clinical Medicine Research 1, no. 4 (2013): 71-74. doi: 10.12691/ajcmr-1-4-6.

\section{Introduction}

Neuroeconomics has been an active research field in the past few decades, aimed at identifying the neural mechanisms of human economic decisions [1,2]. The human decision-making process is complicated and does not always work in the ways predicted by economic theory [3]. In highly complex social environments (e.g., social dilemmas), it must be tailored for that environment and updated continuously with new information as it emerges [4]. The prisoner's dilemma (PD) is a wellstudied economic game analyzed in game theory, originally formulated by Merrill Flood and Melvin Dresher in 1950. The game involves two players, where each can choose between cooperation and defection. Each player receives a higher payoff by defecting, regardless of whether the other player chooses to cooperate or defect, but the payoff to each player is higher for mutual cooperation than for mutual defection, hence creating a dilemma. In the present study, a PD game with an approval stage (PDAS) was used to examine the differences in neural activation without changing the fundamental structure of the PD game. The PDAS is the result of a simple modification to the PD game, whereby an additional stage (the approval stage) is included. In the approval stage, each player can approve or reject the opponent's choice of strategy after playing a PD game. If players mutually approve of each other's choice, the outcome is the same as their original choice. However, if either one rejects the choice of the other, the resulting outcome is the same as when both players defect. The addition of the approval stage resulted in nearly $100 \%$ cooperation in previous economic experiments [5].

With the advent of brain imaging methods such as functional MRI (fMRI), functional near-infrared spectroscopy (fNIRS) and magnetoencephalography (MEG), it became possible to measure the neural correlates of the decision making process directly. fNIRS is an emerging technique which measures hemodynamic variation caused by neural activity in cortical regions. This non-invasive technique utilizes the ability of near-infrared (NIR) light to penetrate through the human scalp and skull to reach the cortex, where it is mainly absorbed by deoxygenated and oxygenated hemoglobin (deoxy-Hb and oxy-Hb, respectively). These two chromophores have distinct absorption coefficients in the NIR region, and changes in their concentrations in the brain can be quantified using at least two wavelengths in this region. fNIRS measures cerebral hemodynamics similarly to fMRI. While the spatial resolution of fNIRS is limited, it has an excellent temporal resolution. Irani et al. [6] indicated that fNIRS may have considerable potential for neurological or psychiatric applications due to its simplicity, portability, and insensitivity to motion artifacts. Hoshi et al. [7] also insisted on the utility of fNIRS in studying the neural substrates of human emotions. They found that some neural pathways related to emotions lie in the prefrontal areas, which are easily accessed by fNIRS. Research in psychiatry can also benefit from fNIRS; for example, Nagoro et al. [8] used fNIRS to identify prefrontal dysfunction in attention-deficit hyperactivity disorder (ADHD). Furthermore, Bae et al. [9] applied 
diffusion tensor-MRI, which is now commonly used for neurological disorders, to investigate dorsolateral prefrontal structures in depression. Some studies examined the relation between economic behavior and brain activity, by using both economic games and brain imaging tools $[10,11]$. However, there are few reports on the differences in brain activation due to the characteristics of the economic games themselves.

The purpose of the study was to detect the relation between activations in the prefrontal area and decisionmaking in the PD and PDAS games, and to discuss the possibility of applying economic games to the clinical field.

\section{Materials and Methods}

\subsection{Subjects}

Twelve healthy right-handed male subjects (mean age 21.5 years, range 20-24 years) participated in the study. Subjects had no history of neurological or other disease. Handedness was measured by the Edinburgh Handedness Inventory before the start of the experiment [12]. Written informed consent was obtained from all subjects before data acquisition. The study was approved by the ethical committee of the Institute of Social and Economic Research (ISER), Osaka University, Osaka, Japan.

\subsection{Experimental Tasks}

The same six payoff matrices with symmetric PD structures were used in both the PD and PDAS games. The PDAS games had variations in status-quo, according with the rewards in the PD games. Subjects sat in front of a display, which showed instructions and payoff matrices, and were instructed to choose cooperation or noncooperation in each trial (see Figure 1). To reduce bias in the game structures, the PD and PDAS games were randomized within sessions. Each trial started with a resting period with a white cross shown in the middle of the screen for 5 seconds, followed by a 10 -second prechoice period which indicated whether the trial had an approval stage or not. Next, a payoff matrix was displayed for 30 seconds (choice stage). Subjects had to choose cooperation or non-cooperation within that 30 seconds. PD trials ended with the choice stage, but in PDAS trials the choice stage was followed by the approval stage. During this stage, participants had 20 seconds to approve or reject their partner's choice. Participants were playing with each other (Figure 1).

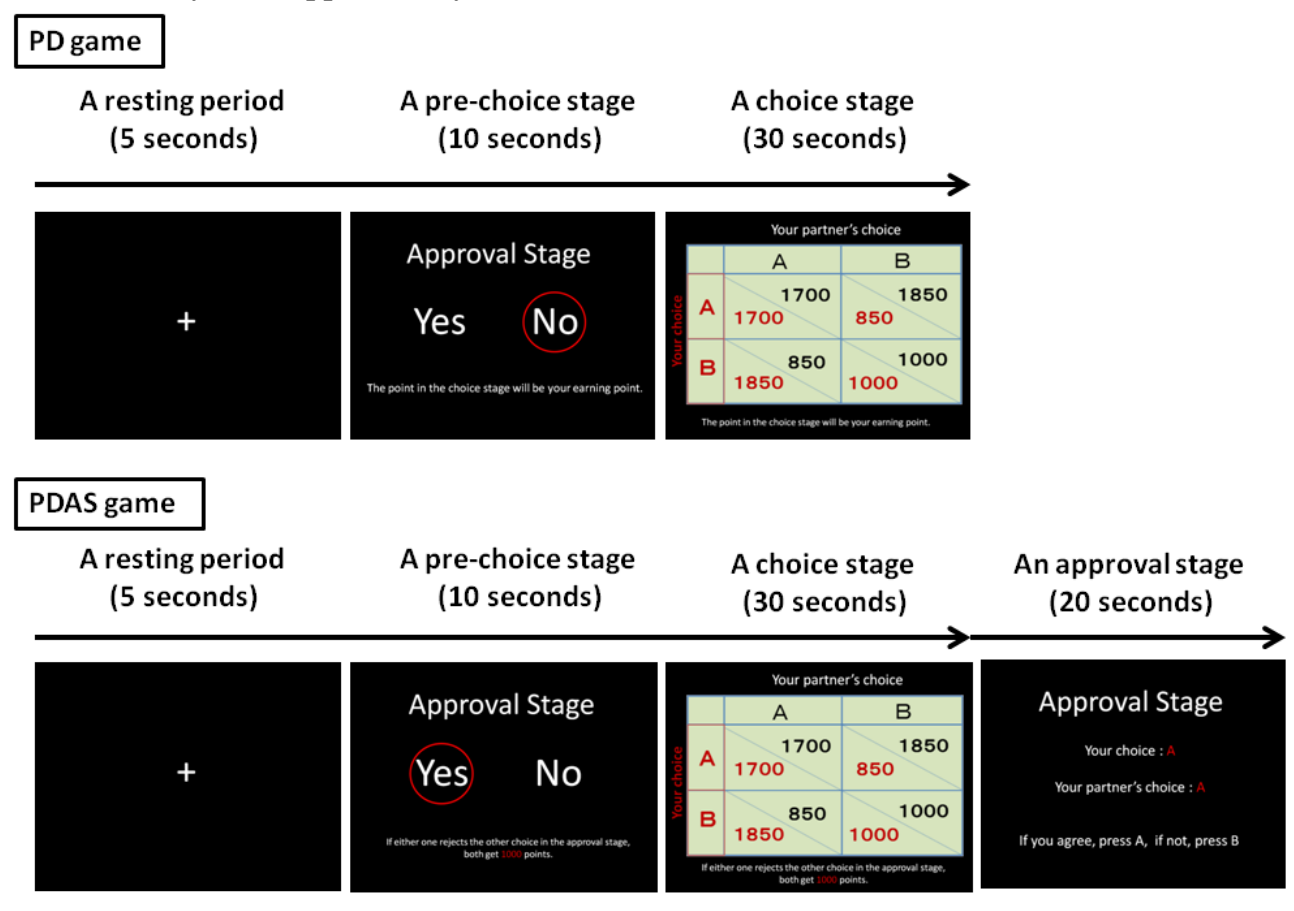

Figure 1. Experimental design. The trials of PD and PDAS games were randomized in the session. The total time of the session was about 10 minutes

\section{3. fNIRS Data Acquisition}

For measuring brain activity, a 45-channel NIRS imaging system (OMM-3000, Oxygenation Multichannel Monitor, Shimazu Co., Kyoto, Japan) was used. Nearinfrared laser diodes with three wavelengths (780, 805, and $830 \mathrm{~nm}$ ) were used as the light sources. The $4 \times 7$ probes were attached over subjects' frontal area. The distance between the emitter and detector probes was set at $3 \mathrm{~cm}$. The lowest probe was positioned along the Fp1Fp2 line in accordance with the International 10/20 System. Changes in the concentration of oxy-Hb and deoxy-Hb were measured with a sampling rate of $10 \mathrm{~Hz}$.
Changes in the concentration of oxy-Hb were analyzed, and NIRS statistical parametric mapping (NIRS-SPM) was used for the group analysis [13].

\section{Results}

All subjects chose cooperation in the PDAS game, but ten out of 12 subjects choose non-cooperation (i.e. defection) in the PD game. Two subjects choose cooperation in the $\mathrm{PD}$ game, despite less reward. Comparisons of oxy-Hb concentrations in the prefrontal area are shown in Figure 2. In ten subjects-those who 
chose defection-there were significant changes in cerebral blood flow in the right prefrontal cortex and left ventromedial prefrontal cortex in the PD game (see Figure 2, A-a), but not in the PDAS game (Figure 2, A-b). In the remaining two subjects, who chose cooperation in both games, no significant change in blood flow was found in either game (Figure 2, B).
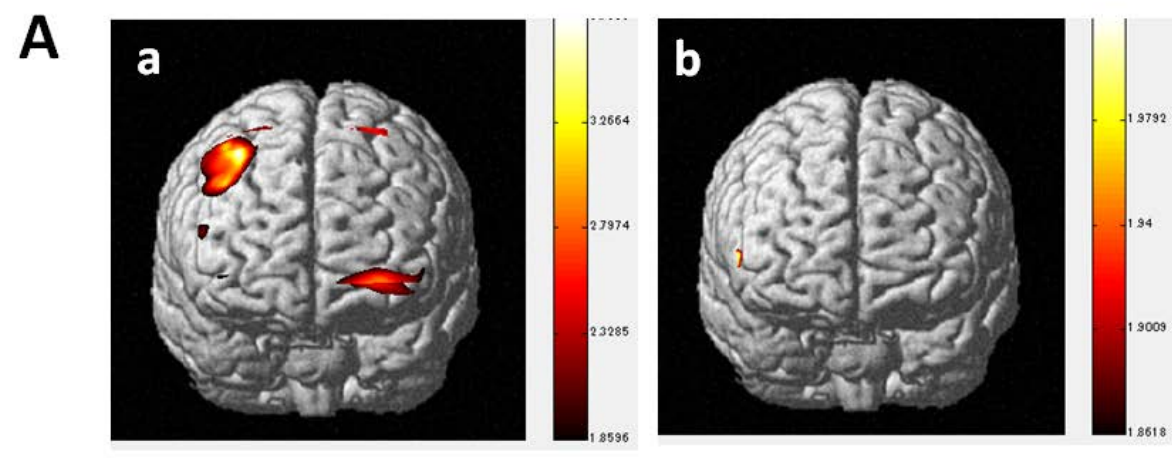

B
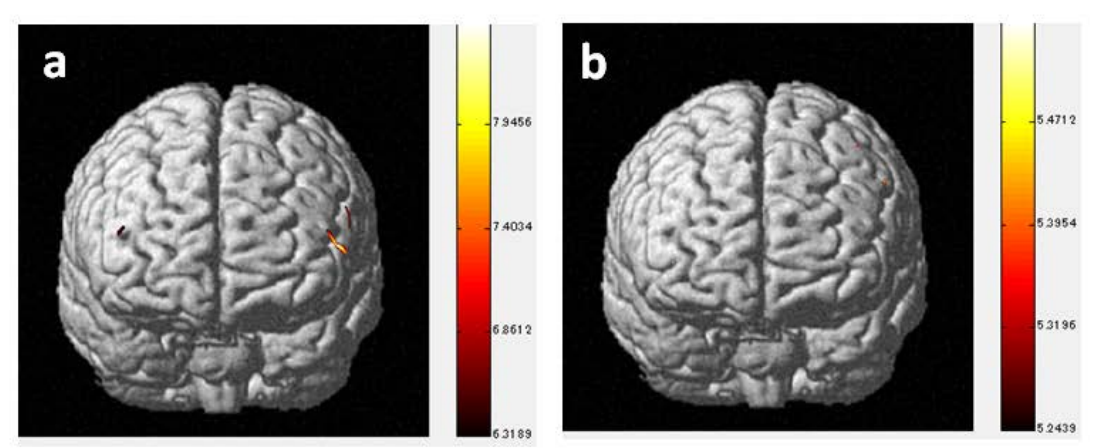

Figure 2. A: Prefrontal regions where significant increases in oxy-Hb concentration were observed in the ten subjects who chose defection in the PD game and cooperation in the PDAS game ( $\mathrm{p}<.05$, corrected). a: The right dorsolateral prefrontal cortex and left orbitofrontal cortex showed significant increases in oxy-Hb concentration during the PD game. b: There was no similar increase in the PDAS game. B: Prefrontal regions of the group analysis in the two subjects who chose cooperation in both the PD and PDAS games ( $<.05$, corrected). There was no significant increase in blood flow in either game. Color bars indicated the threshold of T- statistics

\section{Discussion}

All subjects understood the game structure of the PD and PDAS completely. The structure of the PDAS games made all subjects chose cooperation easily in the decisionmaking. Specifically, Ten out of 12 subjects chose defection in the PD game and cooperation in the PDAS game, while the remaining two subjects chose cooperation in both games. Thus, the addition of the approval stage in the PDAS game induced the majority of participants to change the choices they had made in the PD game. In these subjects, significant activation changes were found in the prefrontal area in the PD game but not in the PDAS game. Lesion studies have suggested that the dorsolateral prefrontal cortex is associated with social cognition processes, including the perception of emotional cues [14], while the ventromedial prefrontal cortex is implicated in dysfunctional real-life social behavior [15]. Hoshi et al [7] also found changes in cerebral blood flow in the lateral prefrontal area related to unpleasant emotions. Our results showed a similar pattern in that there was significant change in the right prefrontal cortex and left orbitofrontal cortex in ten subjects in the PD game. This activation pattern suggests that choosing defection made them experience conflict or some form of unpleasant emotion, which cooperating did not generate. Furthermore, the two subjects who chose cooperation in both games showed no significant changes in blood flow between games-in other words, choosing cooperation does not appear to arouse feelings of conflict.

These results indicate that the characteristics of economic games can easily induce or suppress feelings and change decision-making. In addition, economic games might be a simple way to detect the human mental balance. Therefore, the use of neuroeconomic approaches in the clinical field may provide new ways of identifying the biological substrates of cognitive function and dysfunction.

In conclusion, economic games may provide a way to study cognitive functions using game theory. The combined use of economic games and neuroimaging techniques may capture and quantify neural the substrates of various cognitive processes. They might be effective in extracting human emotional ups and downs in the process of the decision-making. Further studies of economic games for patients with mental illness or brain damage are needed to explore this approach further.

\section{Acknowledgement}

The authors acknowledge Masao Inoue and Yoshiyuki Kato, as well as the Shimadzu Corporation, for their technical support. Furthermore, we also thank Takehito Masuda, a PhD candidate, for his helpful support. This study was supported by the Japan Society for the Promotion of Science (JSPS). 


\section{References}

[1] Glimcher PW, Rustichini A. "Neuroeconomics: the consilience of brain and decision,” Science, 2004. 306(5695). 447-452.

[2] Rustichini A. "Neuroeconomics: what have we found, and what should we search for," Curr Opin Neurobiol, 2009. 19(6). 672-677.

[3] Zak PJ, Kurzban R, Matzner WT. "The neurobiology of trust," Ann N Y Acad Sci, 2004. 1032. 224-227.

[4] Rilling JK, King CB, Sanfey AG. "The neurobiology of social decision-making,” Curr Opin Neurobiol, 2008. 18(2). 159-165.

[5] Saijo T, Okano Y, Yamakawa T. "The Approval Mechanism Experiment: A Solution to Prisoner's Dilemma" in 14th International Conference on Social Dilemmas, 2011.

[6] Irani F, Platek SM, Bunce S, Ruocco AC,Chute D. "Functional near infrared spectroscopy (fNIRS): an emerging neuroimaging technology with important applications for the study of brain disorders," Clin Neuropsychol,2007. 21(1). 9-37.

[7] Hoshi Y, Huang J, Kohri S, Iguchi Y, Naya M, Okamoto T, Ono S. "Recognition of human emotions from cerebral blood flow changes in the frontal region: a study with event-related nearinfrared spectroscopy,” J Neuroimaging,2011. 21(2). e 94-101.

[8] Negoro H, Sawada M, Iida J, Ota T, Tanaka S,Kishimoto T. "Prefrontal dysfunction in attention-deficit/hyperactivity disorder as measured by near-infrared spectroscopy,” Child Psychiatry Hum Dev,2010. 41(2). 193-203.

[9] Bae JN, MacFall JR, Krishnan KR, Payne ME, Steffens DC, Taylor WD. "Dorsolateral prefrontal cortex and anterior cingulate cortex white matter alterations in late-life depression,” Biol Psychiatry,2006. 60(1). 1356-1363.

[10] Nakao T, Osumi T, Ohira H, Kasuya Y, Shinoda J, Yamada J, "Neural bases of behavior selection without an objective correct answer," Neurosci Lett,2009. 459(1). 30-34.

[11] Kishida KT, King CB, Montague PR. "Neuroeconomic approaches to mental disorders,” Neuron, 2010. 67(4). 543-554.

[12] Oldfield RC. "The assessment and analysis of handedness: the Edinburgh inventory,” Neuropsychologia, 1971. 9(1). 97-113.

[13] Ye JC, Tak S, Jan, KE, Jung J,Jang J. "NIRS-SPM: statistical parametric mapping for near-infrared spectroscopy,” Neuroimage, 44(2). 428-447. 2009.

[14] Mah L, Arnold MC,Grafman J. "Impairment of social perception associated with lesions of the prefrontal cortex," Am J Psychiatry,2004. 161(7). 1247-1255.

[15] Krajbich I, Adolphs R, Tranel D, Denburg NL, Camerer CF. "Economic games quantify diminished sense of guilt in patients with damage to the prefrontal cortex," J Neurosci,2009. 29(7). 2188-2192. 